\title{
Molecular profiling and clinico-pathological characteristics of vulvar and vaginal melanomas
} \author{
Mazzarol $^{1}$ \\ ${ }^{1}$ Division of Pathology, European Institute of Oncology, Milan, Italy \\ ${ }^{2}$ Division of Dermato-Oncological Surgery, European Institute of Oncology, Milan, Italy \\ ${ }^{3}$ Division of Epidemiology and Biostatistic, European Institute of Oncology, Milan, Italy \\ ${ }^{4}$ Division of Pathology, IRCCS San Donato, San Donato Milanese, Milan, Italy
}

Caterina Fumagalli ${ }^{1}$, Giulio Tosti $^{2}$, Sara Gandini ${ }^{3}$, Davide Vacirca $^{1}$, Marco Turina $^{4}$, Elena Guerini-Rocco ${ }^{1}$, Massimo Barberis $^{1 *}$ and Giovanni

\begin{abstract}
Vulvar and vaginal melanomas are infrequent malignancies characterized by an aggressive behaviour and a poor prognosis. Surgery is the treatment of choice for primary tumors and loco-regional metastases. As target therapies have emerged as effective treatments both in the advanced and adjuvant settings, molecular profiling of primary tumors and/or metastases is mandatory in order to choose the proper treatment in melanoma patients harbouring specific mutations. Recently, some studies have analyzed the molecular profile of urogenital melanomas, but limited to few specimens and ending with a lack of general agreement. We evaluated a cohort of vulvar and vaginal melanomas for clinical-pathological parameters and molecular profile of the most frequently mutated genes in melanomas, $K I T, B R A F$ and NRAS. Our study population was composed of 23 cases, including 14 vulvar, 7 vaginal and 2 cases of concomitant vulvar and vaginal melanomas. We analyzed clinical-pathological parameters such as histotype, number of mitoses, presence of ulceration, regression, vascular invasion and metastasis, in addition to recurrence and overall survival. Molecular analyses were performed by bi-directional Sanger sequencing for KIT (exons 9,11,13 and 17), BRAF (exons 11 and 15) and NRAS (exons 2 and 3). We applied multivariate models to assess the associations of $K I T, B R A F$ and $N R A S$ status with prognostic factors, adjusting for age. KIT mutations were found in 4 samples (17\%), 3 vulvar and 1 vaginal melanoma. $N R A S$ alterations were observed in 2 of 15 available samples (13\%) equally distributed between the two categories; $B R A F$ mutations were detected in 1 vulvar melanoma of 19 available samples (5\%). KIT mutation was associated with significantly lower number of mitosis and lower Breslow thickness $(\mathrm{P}=0.01$ and $\mathrm{P}=0.05$, respectively, adjusting for age and site). Thicker melanomas were found to be associated with a worse progression free and overall survival $(\mathrm{P}=0.01$ and $\mathrm{P}=0.03$, respectively, adjusting for age). In conclusion in our cohort, melanomas of female genital tract harboured mutations in all the three genes tested, with $K I T$ and $N R A S$ most commonly mutated. KIT status was found to be significantly associated with prognostic factors. Since melanoma patients carrying mutations of $K I T, N R A S$ or BRAF might benefit from target therapy such as imatinib, MEK inhibitor or anti-BRAF drugs, we suggest that even in these rare melanomas molecular analysis should be performed in order to drive proper therapeutic treatment.
\end{abstract}

\section{Introdution}

Vaginal and vulvar melanomas are rare tumors, representing 1-3\% of melanomas arise in women $[1,2]$ and less than $1 \%$ of all female genital tract malignancies [3,4]. Among primary melanomas of the genital tract, vulvar melanomas exhibit the highest frequency, followed by vaginal and cervical. The estimated incidence is $0.1-0.2 / 100.000$ and $0.26-0.46 / 1.000 .000$ new diagnosed cases per year of vulvar and vaginal melanomas, respectively $[1,2,5,6]$. Primary vulvar and vaginal melanomas more frequently occur in Caucasian women in their fifth to seventh decade of life [7-10].

The overall prognosis is poor, with a 5 -year survival rate of approximately $47 \%$ for primary vulvar melanoma [1] and $18 \%$ for primary vaginal melanoma [1,7]. Loco-regional control and survival rates are reported to be related to tumor thickness, ulceration, lymph node spread and age $[8,11]$. The treatment of choice is surgery [12], but there is lack of consensus about the extent of the surgical approach (i.e., conservative wide local excision versus radical surgery) and the role of the sentinel node biopsy for microscopic staging. The benefit of adjuvant therapies for higher-risk (i.e., American Joint Committee on Cancer (AJCC) stage II or III) melanomas after surgical excision needs to be further investigated $[13,14]$. Due to the rarity of these tumors and the lack of evidence-based guidelines for the management of vulvar and vaginal melanoma, these patients are usually treated in accordance to principles of management of cutaneous melanoma [14-16].

Cutaneous melanoma molecular characterization is mainly focused on the MAPK/ERK pathway, with BRAF mutations found in up to $55 \%-60 \%$ of primary tumors arising on intermittently sunexposed skin, without sun-induced damage $[17,18]$. However, vulvar and vaginal primary melanomas show a lower rate of $B R A F$ mutations (less than 15\%) [17,18] but harbor KIT mutations in up to $40 \%$ [19] and NRAS mutations in up to $14 \%$ of cases [20]. Recently, some studies on vulvo-vaginal melanomas have shown that KIT aberrations are the most common alterations in vulvar melanomas, while NRAS mutations are more frequently observed in vaginal melanomas [21-25]. Due to the limited number of samples tested in each study and the difficult labelling among vulvar or vaginal category, these findings need to be further investigated.

Correspondence to: Massimo Barberis, MD, Division of Pathology, European Institute of Oncology, via G. Ripamonti 435, 20141, Milan, Italy, Tel: 02577489414; E-mail: Massimo.Barberis@ieo.it

Key words: Vulvo-vaginal melanomas, BRAF, KIT, NRAS

Received: May 02, 2016; Accepted: May 19, 2016; Published: May 23, 2016 
Nowadays there are active therapies targeting altered genes such as BRAF, KIT or NRAS, with promising results from clinical trials enrolling a subset of patients harboring specific alteration [26-36]. Therefore, extended studies should be performed recruiting vulvovaginal melanoma patients, to deeply understand the molecular profile of these lesions, in order to identify patients who may benefits from targeted therapies.

The aim of our study was to evaluate the pathological and molecular features of malignant melanomas of the female genital tract, carefully subdivided into vulvar or vaginal site of onset, in association with prognostic factors and overall survival, in our single-center cohort. We focused on KIT, BRAF and NRAS which are the current targets of molecular therapies in melanomas.

\section{Materials and methods}

\section{Patient's selections}

In this study, we evaluated 23 patients with primary vulvar or vaginal melanoma who were diagnosed and treated between 1999 and 2012 at the Istituto Europeo di Oncologia, Milan, Italy. The cohort of patients included 14 vulvar, 7 vaginal and 2 patients with a concomitant vulvar and vaginal primary melanomas. The histopathological classification was performed by two experienced dermatopathologists according to WHO guidelines, evaluating Breslow's tumor thickness, number of mitoses, presence of ulceration, regression, vascular invasion and metastasis (Figure 1 and Table 1). Consensus was achieved in case of discordance. Complete follow-up was available for 22 patients. All the patients underwent surgical resection with intention to treat; none received target therapies which were not available at the time of diagnosis.

\section{Mutational analyses}

KIT (exons 9,11,13 and 17), BRAF (exons 11 and 15) and NRAS (exons 2 and 3) mutational analysis was performed by bi-directional
Sanger sequencing. Briefly, the pathologist identified tumor areas with a tumor cellular content of at least $60 \%$; sections of $5-\mu \mathrm{m}$ thickness were cut from paraffin blocks and the previously identified areas were macrodissected by a sterile scalpel. DNA was extracted using a commercial available kit (QIAamp DNA FFPE Tissue kit, Qiagen, Hiden, Germany), amplified and then purified by enzyme treatment with Exonuclease I and Shrimp Alkaline Phosphatase (GE Healthcare Life Sciences, Little Chalfont, Buckinghamshire, UK). The cycle sequencing reactions were carried out using BigDye Terminator chemistry, followed by removing of unincorporated reagents with BigDye XTerminator kit (Applied Biosystems, Foster City, CA). Sequencing reaction products underwent capillary electrophoresis using 3500Dx Genetic Analyzer (Applied Biosystems, Foster City, CA) and was sequenced on both forward and reverse directions. Mutations were confirmed in two independent amplification runs.

According to the division policy and in order to save tissue, a diagnostic algorithm was applied considering the frequency of the mutations. KIT was the first gene studied and the wild-type cases were subsequently investigated for $B R A F$ and NRAS gene status. In mutated cases, no further analysis was performed due to the mutual exclusivity of these mutations.

\section{Statistical analysis}

Descriptive statistics were used to analyze tumor and patients characteristics. Data were reported as relative frequencies (percentage) for categorical variables and median values and interquartile ranges for continuous variables.

We investigated the association of KIT, BRAF and NRAS gene status with melanoma prognostic factors through univariate analyses with Chi-square tests and Wilcoxon rank tests and through multivariate linear models (analysis of covariance, ANCOVA) and linear logistic models, to verify the associations adjusting for confounders.

Overall survival (OS) was calculated from time of diagnosis to

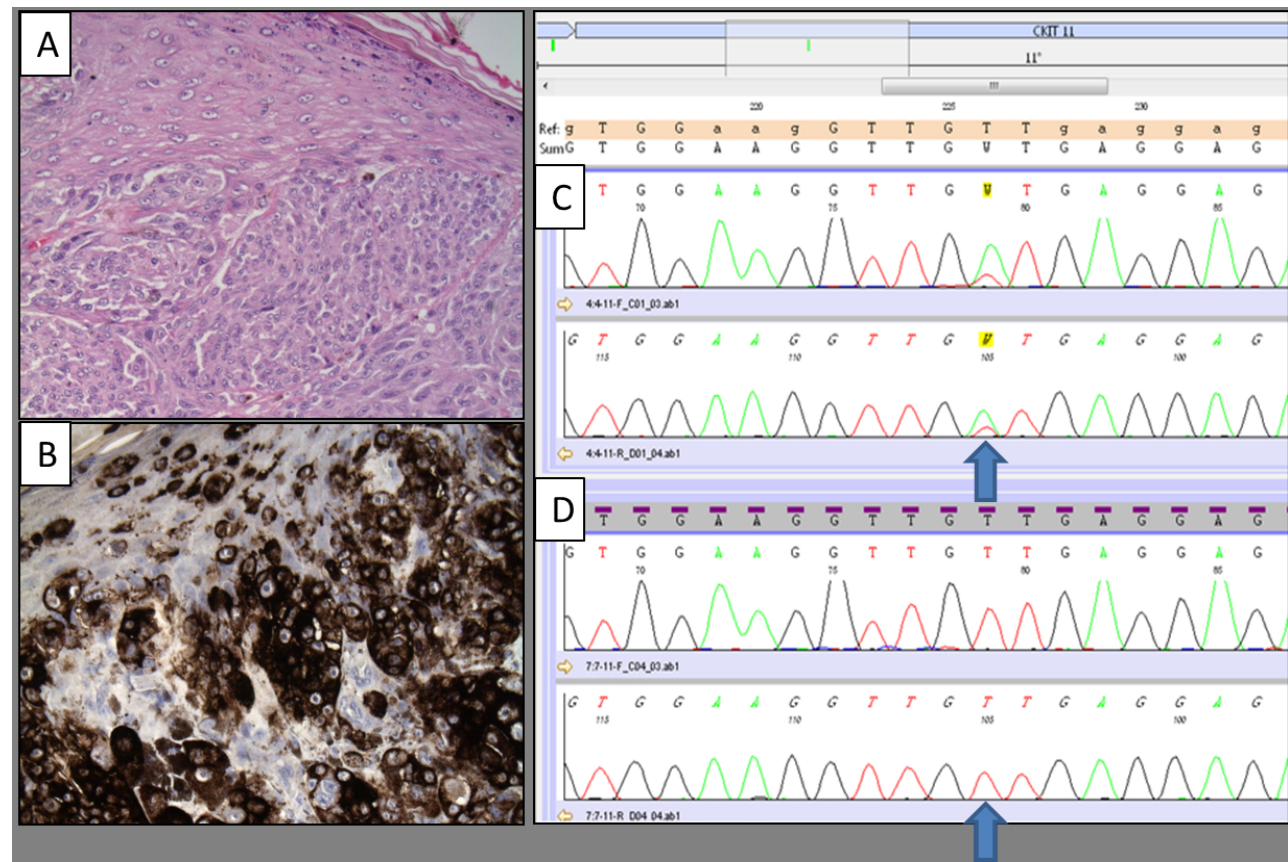

Figure 1. Micrographs showing a detail of vulvar melanoma (A; hematoxylin and eosin stain, original magnification: 200x) displaying strong HMB-45 expression (B; immunohistochemical stain, original magnification: 200x). The mutational analysis of the same cases revealed the presence of KIT exon $11 \mathrm{p}$.V560D mutation (C; electropherogram image obtained by Bidirectional Sanger Sequencing). Electropherogram image of wild-type sequence of the same gene is reported for comparison (D). 
Table 1. Patients and tumor characteristics.

\begin{tabular}{|c|c|c|c|}
\hline & & Frequency & $\%$ \\
\hline Age (Median, q1-q3*) & $71(57-76)$ & & \\
\hline Mitosis (Median, q1-q3*) & $12(6-15)$ & & \\
\hline Breslow (Median, q1-q3*) & $5(3-9)$ & & \\
\hline \multirow[t]{3}{*}{ Site } & Vagina & 7 & 30 \\
\hline & Vulva & 14 & 61 \\
\hline & Vulva and vagina & 2 & 9 \\
\hline \multirow[t]{5}{*}{ Histological type } & $\begin{array}{l}\text { Mucous lentiginous } \\
\text { melanoma }\end{array}$ & 2 & 9 \\
\hline & Nodular melanoma & 3 & 13 \\
\hline & $\begin{array}{l}\text { Superficial spreading } \\
\text { melanoma }\end{array}$ & 12 & 52 \\
\hline & Polipoid melanoma & 1 & 4 \\
\hline & Missing & 5 & 22 \\
\hline \multirow[t]{3}{*}{$B R A F$} & $\mathrm{WT} \dagger$ & 18 & 78 \\
\hline & $\mathrm{pV} 600$ & 1 & 4 \\
\hline & Missing & 4 & 18 \\
\hline \multirow[t]{4}{*}{ KIT } & $\mathrm{WT} \dagger$ & 19 & 83 \\
\hline & p.V560D & 2 & 9 \\
\hline & p.Y578H & 1 & 4 \\
\hline & p.N655K & 1 & 4 \\
\hline \multirow[t]{3}{*}{$N R A S$} & $\mathrm{WT} \dagger$ & 13 & 56 \\
\hline & p.Q61R & 2 & 9 \\
\hline & Missing & 8 & 35 \\
\hline \multirow[t]{3}{*}{ Ulceration } & No & 6 & 26 \\
\hline & Yes & 16 & 70 \\
\hline & Missing & 1 & 4 \\
\hline
\end{tabular}

*q1-q3: range interquartile; †WT: wild-type

death or last follow-up (censored) and Progression Free Survival (PFS) from time of diagnosis to first event (progression or death) or last follow-up (censored). OS curves were estimated with the Kaplan-Meier method. Regression Cox models were used to assess the association of $K I T, B R A F$ and NRAS gene status and prognostic factors with OS or PFS adjusting for confounders.

Since the relative small size of the sample we decided to present P-values from simple multivariate models adjusted for age only. Including more confounders would make models unstable.

For all analyses, two-tailed $\mathrm{P}<0.05$ was considered statistically significant. The statistical analyses were performed with the Statistical Analysis System Version 9.2 (SAS Institute, Cary, NC).

\section{Results}

Clinical and pathological features of the overall study cohort $(n=23)$ are summarized in Table 1 . The cohort included 14 patients with primary vulvar melanoma, 7 patients with primary vaginal melanoma and 2 patients with a synchronous vulvar and vaginal melanoma.

\section{Pathological features}

Data on melanoma histotype were available for 18 patients (Table 1). Superficial spreading melanoma was the most common histotype (12 cases, $66.6 \%)$, followed by nodular melanoma ( $3 ; 16.6 \%)$, mucous lentiginous melanoma $(2 ; 11.1 \%)$ and 1 case of polipoid melanoma (5.5\%).

Data on Breslow thickness, presence of ulceration and number of mitosis were available for all but one vulvar melanomas $(n=22)$. Median Breslow thickness for the whole cohort was $5 \mathrm{~mm}$ with interquartile range: 3 to $9 \mathrm{~mm}$. The vulvar cohort has a median Breslow thickness of
$4.5 \mathrm{~mm}$ (interquartile range: 2.3 to $5 \mathrm{~mm})$, significantly $(\mathrm{P}=0.02)$ lower than the vaginal cohort (median Breslow: $9 \mathrm{~mm}$, interquartile range: 5 to $15 \mathrm{~mm}$ ). Breslow thickness was associated with progression free survival and overall survival ( $\mathrm{P}=0.01$ and 0.03 ; adjusting for age and site) (Figure 2). Sixteen out of 22 primary vulvar and vaginal melanomas were ulcerated $(72.8 \%)$. Median number of mitosis per square $\mathrm{mm}$ for the whole cohort of primary vulvar and vaginal melanomas was 11.5 with interquartile range of 6 to 15 (median number: 12 and 10 , interquartile range 6-25 and 7-15 for vulvar and vaginal melanomas, respectively).

\section{Surgical treatment of the primary tumor}

Patients with primary vulvar melanoma $(n=14)$ received the following treatments: radical vulvectomy $(n=5)$, hemivulvectomy $(\mathrm{n}=4)$ and wide local excision $(\mathrm{n}=5)$. Patients with a primary vaginal melanoma $(n=7)$ received vaginectomy $(n=3)$, vaginectomy and hysterectomy $(n=3)$ and exenteratio $(n=1)$. The two patients with synchronous vulvar and vaginal melanoma received hemivulvectomy and vaginectomy in one case and hemivulvectomy, vaginectomy and hysterectomy in the other case.

\section{Lymph node status management}

Sentinel lymph node biopsy was performed in 9 out 14 patients with primary vulvar melanoma. Three positive patients underwent therapeutic node dissection. Five patients with primary vulvar melanoma had no sentinel lymph node biopsy and 4 of them had an elective lymph node dissection. Three out of these 4 cases had confluent metastatic nodes or more than seven metastatic lymph nodes. One patient refused any further treatment after the excision of the primary vulvar melanoma. Five out of 7 patients with primary vaginal melanoma and 2 cases of concomitant vulvar and vaginal melanoma underwent elective lymph node dissection.

\section{Follow-up events}

Median follow-up was 4.1 years. Data on loco-regional recurrence were retrieved for 22 out of 23 patients included in the study. Fourteen

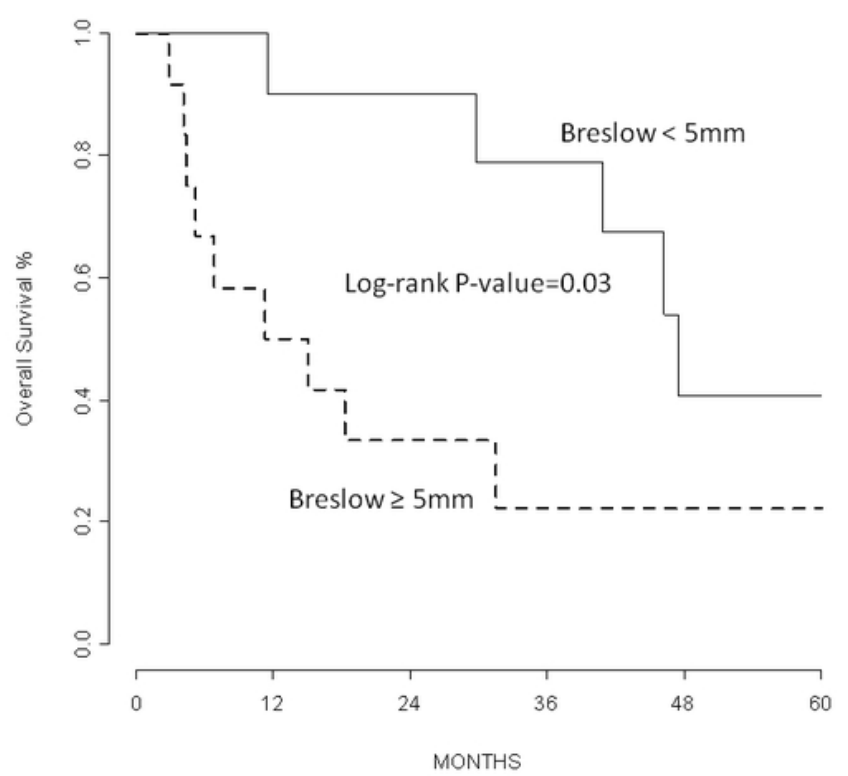

Figure 2. Kaplan-Meier curves show that Breslow thickness was associated with overall survival $(\mathrm{P}=0.03$; adjusting for age and site). 
patients (63.6\%) developed at least one regional event during followup. Regional event was defined as melanoma recurrence at the genitourinary tract or at the regional lymph nodes after radical treatment of primary tumor. Other 4 patients developed distant metastases. In 10 patients loco-regional events preceded the onset of distant metastases. Sixteen out of 23 patients died for the disease, with an overall median survival of 41 months (95\%CI: 11-64), and of 45 (95\%CI: 11-100) and 7 months (95\%CI: 3-30), for vulvar and vaginal melanoma, respectively.

\section{Molecular profile}

All cases were screened for KIT alterations and 4 specimens (3 vulvar melanoma and 1 vaginal melanoma) harbored KIT mutation (17\%) (Figure 1). NRAS alterations were observed in 2 of the 15 available samples (13\%), including 1 case of primary vulvar melanoma and 1 case of primary vaginal melanoma. BRAF mutation was detected in 1 vulvar melanoma (5\%) among the 19 vulvo-vaginal cases analyzed. KIT mutations were located in exon 11 (codons 560 and 578) and exon 13 (codon 655), while NRAS and BRAF alterations involved exon 2 (codon 61) and exon 15 (codon 600), respectively (Table 1).

In two mutated cases, tissue from recurrence was available, including one loco-regional recurrence and one lymph node metastasis that harbored BRAF and NRAS mutations, respectively. Both specimens maintained the very same mutation detected in the primary tumors.

In our series, mutations of KIT showed a significant association $(\mathrm{P}=0.03)$ with patient age at diagnosis. Patients with KIT mutation had significant older age (78 vs. 67 years). No association was found with clinical stage, histology, ulceration or site of onset. KIT mutation was found to be associated with significantly lower number of mitoses $(\mathrm{P}=0.05$, adjusting for age and site; Table 2$)$ and with a significantly lower Breslow thickness ( $\mathrm{P}=0.05$ adjusting for age and site; Table 3). BRAF and NRAS mutations were not found to be significantly associated with prognostic factors nor follow-up events.

\section{Discussion}

In this study wwe evaluated the mutational status of three drugable genes (KIT, BRAF, NRAS) in a single centre cohort of genital

Table 2. Frequencies of patients by number of mitoses and gene status.

\begin{tabular}{|c|c|c|c|c|}
\hline & & \multicolumn{2}{|c|}{ n. of Mitosis } & \multirow[b]{2}{*}{ P-value* } \\
\hline & & $\leq 6$ & $>6$ & \\
\hline \multirow[t]{4}{*}{ KIT } & WT $\dagger$ & 3 & 15 & 0.05 \\
\hline & & $50 \%$ & $94 \%$ & \\
\hline & p.V560D, p.Y578H, p.N655K & 3 & 1 & \\
\hline & & $50 \%$ & $6 \%$ & \\
\hline \multirow[t]{6}{*}{$B R A F$} & WT† & 5 & 12 & 0.99 \\
\hline & & $83 \%$ & $75 \%$ & \\
\hline & p.V600 & 0 & 1 & \\
\hline & & $0 \%$ & $6 \%$ & \\
\hline & Missing & 1 & 3 & \\
\hline & & $17 \%$ & $19 \%$ & \\
\hline \multirow[t]{6}{*}{$N R A S$} & $\mathrm{WT} \dagger$ & 4 & 9 & 0.55 \\
\hline & & $67 \%$ & $56 \%$ & \\
\hline & p.Q61R & 0 & 1 & \\
\hline & & $0 \%$ & $6 \%$ & \\
\hline & Missing & 2 & 6 & \\
\hline & & $33 \%$ & $38 \%$ & \\
\hline
\end{tabular}

*P-value from logistic model adjusted for age and site; $†$ WT: wild-type
Table 3. Median and interquartile range of Breslow thickness by gene status.

\begin{tabular}{|l|l|c|c|c|c|c|}
\hline \multirow{2}{*}{ KIT } & WT $\dagger$ & N & $\begin{array}{c}\text { Median } \\
\text { Breslow }\end{array}$ & $\begin{array}{c}\text { Lower } \\
\text { Quartile }\end{array}$ & $\begin{array}{c}\text { Upper } \\
\text { Quartile }\end{array}$ & P-value* \\
\cline { 2 - 8 } & p.V560D, p.Y578H, p.N655K & 4 & 3 & 2 & 3.8 & \\
\hline \multirow{3}{*}{$B R A F$} & WT $\dagger$ & 17 & 5 & 4 & 8 & 0.64 \\
\cline { 2 - 8 } & pV600 & 1 & 2.1 & & & \\
\cline { 2 - 8 } & Missing & 4 & 7.2 & 1.7 & 12 & \\
\hline \multirow{2}{*}{ WRAS } & WT $\dagger$ & 13 & 4.5 & 3 & 8 & 0.86 \\
\cline { 2 - 8 } & p.Q61R & 1 & 9 & & & \\
\cline { 2 - 8 } & Missing & 8 & 5 & 2 & 8.5 & \\
\hline
\end{tabular}

*P-value from ANCOVA model adjusted for age and sites; $†$ WT: wild-type

melanomas. The frequencies of KIT mutations detected in our series were 4/23 (17\%) melanomas, 3 arose in vulva and 1 in vagina. Omholt et al. found KIT mutations in 35\% of vulvar melanomas; however, no KIT mutations were found in the 7 vaginal melanomas analyzed [21]. Recently, both Aulmann [22] and Rouzbahman [23] described KIT mutations in $18 \%$ and $27.6 \%$ of vulvar melanomas respectively, but in none of vaginal melanomas tested. Even if KIT mutation appears to be a peculiar sign of vulvar onset, other studies have found mutations even in 1 of 4 [24] and 1 of 24 vaginal melanomas [25]. These differences might be due to the fact that categorizing vulvar versus vaginal melanomas on the basis of the site of onset is demanding and different molecular studies have classified vulvar and vaginal melanomas together as vulvo-vaginal [37], urogenital [38] and genital melanomas [39]. In out cohort, KIT mutated status was associated (6 mitosis cut-off) and lower Breslow's thickness (5 mm cut-off). Since a greater Breslow thickness was associated with a significantly worse prognosis (Figure 2), we could speculate that KIT mutated melanomas might have a better prognosis than KIT wild-type cases. However, due to the small sample size, we did not find any statistically significant evidence of a link between KIT mutational status and other clinical parameters, such as lymph node status, distant metastases occurrence and overall survival, according to the findings of Omholt el al. [21].

Different studies have reported on the presence of NRAS mutation in vaginal melanomas with a wide frequency range: $2 / 15$ (13.3\%) [22], $3 / 7(42.9 \%)$ [21], 3/14 (21.4\%) [25]. However, these data lead to a significantly higher proportion of NRAS mutated vaginal melanomas than mucosal melanomas of other sites [21]. In our cohort, NRAS mutation was detected in one primary vaginal melanoma specimen and in one vaginal recurrence of a primary vulvar melanoma, and accounts for $13 \%(2 / 15)$ of the all cases. Moreover, we found only one $B R A F$ mutation (5\%), according to the studies describing few BRAF mutations in genital lesions [40].

To the best of our knowledge, this is the first report of the presence of genes mutations in primary vulvo-vaginal melanomas that were maintained in the subsequent metastatic or recurrence site. These data were already reported for other site of onset. Although detected on a limited number of patients, these findings highlighted the importance of these genes as neoplastic progression drivers in this subset of melanomas and possible drugable targets.

The cumulative frequency of oncogenic mutations in melanomas of the female genital reported in our series corresponds to the one that has been proposed by Tseng et al. [41]. Although, other studies have showed a lower frequency of mutations, mainly of KIT gene [42], these discrepancies might be related to the small number of cases analyzed and to the different analytical assays used. 
However, regardless the actual frequency of these mutations, the presence of $B R A F, K I T$ and NRAS gene aberrations could be targeted by specific pharmacological inhibitors. Several studies have showed that the molecular characterization allowed the selection of patients who could benefit from target therapies. The type of KIT mutation and the ratio of mutant to wild-type KIT alleles were predictive of clinical response to imatinib $[27,28]$. Moreover other drugs such as dasatinib [29] and sunitinib [30] and nilotinib [31] are under investigation in phase II clinical trials for KIT mutated patients. The clinical relevance of BRAF inhibitors in treatment of melanoma patients carrying $B R A F$ codon 600 mutations is well known [32,33] and new studies highlight the importance of a combination with MEK inhibitors [34]. Ascierto et al. reported that binimetinib have shown a robust activity specifically in NRAS mutated melanoma [35] and new clinical trials are enrolling NRAS mutated patients [36]. Unfortunately, no molecular target therapy was administered to any patients investigated in this study and no further data on treatment response and survival are available.

Therefore, since vulvo-vaginal melanoma could harbor KIT, NRAS, $B R A F$ mutations (overall $30 \%$ in our study), which are also maintained in the recurrent/metastatic disease, molecular analyses need to guide current therapeutic choices for these patients. However, further trials and multicenter studies are required to better understand the clinical impact of target therapy in urogenital melanomas.

\section{Autorship}

Conception and design: Caterina Fumagalli, Massimo Barberis, Giovanni Mazzarol; Acquisition of data: Caterina Fumagalli, Giulio Tosti, Sara Gandini, Davide Vacirca, Marco Turina, Giovanni Mazzarol; Analysis and interpretation of data: Caterina Fumagalli, Giulio Tosti, Sara Gandini, Davide Vacirca, Marco Turina, Elena Guerini-Rocco, Massimo Barberis, Giovanni Mazzarol; Writing, review, and/or revision of the manuscript: Caterina Fumagalli, Giulio Tosti, Sara Gandini, Davide Vacirca, Marco Turina, Elena GueriniRocco, Massimo Barberis, Giovanni Mazzarol.

\section{Acknowledgment}

Laboratory technicians of division of pathology, European Institute of Milan.

\section{References}

1. Dubowitz V, Brooke MH (1973) Muscle biopsy: a modern approach. WB Saunders, London, UK.
2. Dubowitz V (1986) Muscle biopsy: a practical approach. (2ndEdtn). BaillièreTindall, London, UK.

3. Helge JW, Rehrer NJ, Pilegaard H, Manning P, Lucas SJ, et al. (2007) Increased fat oxidation and regulation of metabolic genes with ultraendurance exercise. Acta Physiol (Oxf) 191: 77-86. [Crossref]

4. Jeukendrup AE, Moseley L, Mainwaring GI, Samuels S, Perry S, et al. (2006) Exogenous carbohydrate oxidation during ultraendurance exercise. J Appl Physiol 100: 1134-1141. [Crossref]

5. Alghannam AF, Jedrzejewski D, Bilzon J, Thompson D, Tsintzas K, et al. (2016) Influence of Post-Exercise Carbohydrate-Protein Ingestion on Muscle Glycogen Metabolism in Recovery and Subsequent Running Exercise. Int J Sport Nutr Exerc Metab. [Crossref]

6. Hardie DG, Carling D, Carlson M (1998) The AMP-activated/SNF1 protein kinase subfamily: metabolic sensors of the eukaryotic cell? Annu Rev Biochem 67: 821-855. [Crossref]

7. Chen ZP, McConell GK, Michell BJ, Snow RJ, Canny BJ, et al. (2000) AMPK signaling in contracting human skeletal muscle: acetyl-CoA carboxylase and NO synthase phosphorylation. Am J Physiol Endocrinol Metab 279: E1202-1206. [Crossref]

8. Derave W, Ai H, Ihlemann J, Witters LA, Kristiansen S, et al. (2000) Dissociation of AMP-activated protein kinase activation and glucose transport in contracting slowtwitch muscle. Diabetes 49: 1281-1287. [Crossref]

9. Fujii N, Hayashi T, Hirshman MF, Smith JT, Habinowski SA, et al. (2000) Exercise induces isoform-specific increase in 5'AMP-activated protein kinase activity in human skeletal muscle. Biochem Biophys Res Commun 273: 1150-1155. [Crossref]

10. Jørgensen SB, Viollet B, Andreelli F, Frøsig C, Birk JB, et al. (2004) Knockout of the alfa-2 but not alfa-1 5'-AMP-activated protein kinase isoform abolishes 5-aminoimidazole-4-carboxamide-1-beta-4-ribofurasonide but not contraction-induced glucose uptake in skeletal muscle. J Biol Chem 279: 1070-1079. [Crossref]

11. Frayn KN (2003) “The Glucose/Fatty Acid Cycle 1963-2003: A Tribute to Sir Philip Randle”. The glucose-fatty acid cycle: physiological perspective. Biochemical Society Transactions 31: 1115-1119.

12. Tyler CJ, Reeve T, Hodges GJ, Cheung SS (2016) The Effects of Heat Adaptation on Physiology, Perception and Exercise Performance in the Heat: A Meta-Analysis. Sports Med. [Crossref]

13. Adeva-Andany MM, González-Lucán M, Donapetry-García C, Fernández-Fernández C, Ameneiros-Rodríguez E (2016) Glycogen metabolism in humans. Biochimicaet biophysicaacta clinical 5: 85-100. [Crossref]

14. Shearer J, Graham TE (2004) Novel aspects of skeletal muscle glycogen and its regulation during rest and exercise. Exerc Sport Sci Rev 32: 120-126. [Crossref]

15. Rose AJ, Richter EA (2005) Skeletal muscle glucose uptake during exercise: how is it regulated? Physiology (Bethesda) 20: 260-270. [Crossref]

Copyright: (C2016 Fumagalli C. This is an open-access article distributed under the terms of the Creative Commons Attribution License, which permits unrestricted use, distribution, and reproduction in any medium, provided the original author and source are credited. 